\title{
Idiopathic pulmonary fibrosis and systemic sclerosis: pathogenic mechanisms and therapeutic interventions
}

\author{
Hamid Mattoo ${ }^{1} \cdot$ Shiv Pillai ${ }^{2}$ D \\ Received: 25 December 2020 / Revised: 7 May 2021 / Accepted: 5 June 2021 / Published online: 18 June 2021 \\ (c) The Author(s), under exclusive licence to Springer Nature Switzerland AG 2021
}

\begin{abstract}
Fibrotic diseases take a very heavy toll in terms of morbidity and mortality equal to or even greater than that caused by metastatic cancer. In this review, we examine the pathogenesis of fibrotic diseases, mainly addressing triggers for induction, processes that lead to progression, therapies and therapeutic trials. For the most part, we have focused on two fibrotic diseases with lung involvement, idiopathic pulmonary fibrosis, in which the contribution of inflammatory mechanisms may be secondary to non-immune triggers, and systemic sclerosis in which the contribution of adaptive immunity may be predominant.
\end{abstract}

Keywords Mechanisms of fibrosis · Lung fibrosis · Risk factors · Epithelial cell homeostasis

\section{Introduction: fibrosis reflects the loss of homeostasis resulting in excessive tissue repair}

Fibrosis is a pathological term describing the excessive accumulation of extracellular matrix in a tissue. This process typically results from tissue injury followed by unregulated and overexuberant repair. The replacement of functioning cells and the induction of disordered tissue architecture induced by collagen-rich "scars" typically compromises organ function [1, 2]. Organs such as the lung, liver, kidney, the gastrointestinal tract, and skin are composed of epithelial cells as well as many other different cell types and are exposed to different environmental factors. Fibrosis and the complications that result from fibrotic diseases contribute to nearly $45 \%$ of all deaths in the United States.

A dysfunctional, often overexuberant, repair process following injury results in different fibrotic disease phenotypes unique to each of these organs. Injury can be initiated by identifiable triggers such as infection and cancer, but most often the inciting triggers that lead to the evolution of a fibrotic disease are not known and likely

Shiv Pillai

pillai@helix.mgh.harvard.edu

1 Immunology and Inflammation Research Therapeutic Area, Sanofi, Cambridge, MA, USA

2 Ragon Institute of MGH, MIT and Harvard, Cambridge, MA, USA differ from organ to organ and from disease to disease. However, when considering subsequent events, there are certain common features in different fibrotic diseases that are shared across different organs [1]. In general, injury or death of parenchymal cells (typically epithelial cells) in a tissue results in the activation of innate immunity, this is followed by some regulated tissue remodeling and then homeostasis is restored (Fig. 1). In a fibrotic disease, there is typically a persistent source of tissue injury or apoptotic death that contributes to uncontrolled tissue remodeling usually in the context of uncontrolled or persistent inflammation as well (Fig. 1). It is helpful, though this may be an oversimplification, to think of fibrosis as having temporal and interconnected stages. The initial or triggering stage typically involves epithelial injury and dysfunction. This is followed by a stage of immune cell recruitment, though the degree of inflammation in different disorders may vary, and indeed immune cells may themselves serve as the "trigger" in some disorders. This could occur when immune cells are autoreactive or because the parenchymal cells are infected and thus serve as immunological targets. Fibrosis culminates with a final stage of dysregulation within the mesenchyme, those portions of an organ that are often described as "connective tissue". This final stage may be propagated and amplified by tissue hypoxia, tissue rigidity or stiffness and epigenetic alterations in innate immune cells [2, 3]. Understanding the nuances of the pathogenic processes that are common to different fibrotic diseases can help 


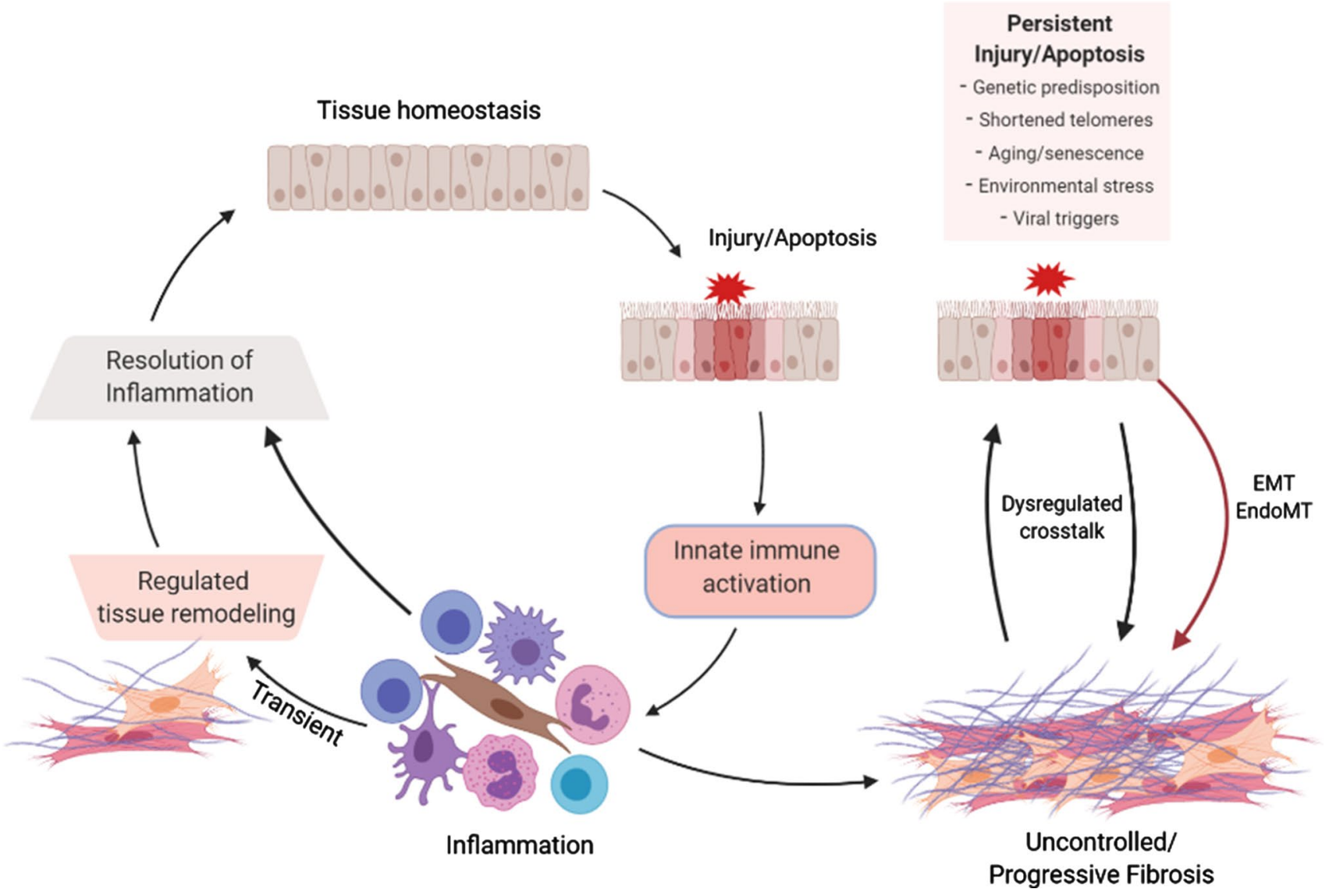

Fig. 1 An overview of the pathogenic events in fibrosis

us identify druggable pathways and molecules that could be targeted using a plethora of modern drug development technologies.

Notions of what drives fibrosis are continuing to evolve, and it is our lack of precise knowledge that in part contributes to the lack of effective therapies for these diseases. While reversing previous scarring of a tissues is a challenge, the goal of therapy is to not just prevent ongoing cellular and molecular events that continue to drive the process of fibrosis, but also induce tissue regeneration by skewing the balance of some well-understood pathogenic mechanisms.

In this review, we will first briefly touch upon two debilitating fibrotic disease both of which involve the lung but have distinct features. Through the lens of these two diseases, we will summarize distinct, but yet to be firmly established, initiating mechanisms that may lead to fibrosis and then discuss the stages of perpetuation and progression of fibrosis shared between these two diseases. We will end with some thoughts about newer systems approaches in these two diseases to identify novel therapeutics that might come to fruition in the not-too-distant future.

\section{Section one: human fibrotic diseases}

In this section, we touch on two debilitating fibrotic diseases, idiopathic pulmonary fibrosis (IPF) and Systemic sclerosis (SSc), both of which target the lung, to illustrate the mechanistic understanding of fibrogenesis in human diseases. IPF and SSc-ILD (interstitial lung disease in systemic sclerosis) are distinct lung diseases in which there is still much more to be learnt about the biological bases for disease presentation and this knowledge could impact clinical diagnosis and patient management [3]. Although both these conditions present with dysregulated fibroblast activation and myofibroblast accumulation, the initiating events and pathways of disease perpetuation and progression appear to be vastly distinct. While persistent alveolar and airway epithelial injury defines the core of IPF disease pathogenesis, SSc-ILD manifests defective endothelial cell homeostasis [4]. Unlike in IPF where the role of inflammation in disease pathogenesis and progression has been increasingly questioned recently, SSc and SSc-ILD are well documented to have a major inflammatory component [5]. 


\section{Idiopathic pulmonary fibrosis}

IPF is a chronic, progressive form of interstitial lung disease with an overall prevalence of 5-30 cases per 100,000 per year and a median survival rate of 3-5 years post-diagnosis (with no disease intervention) [6]. IPF is a diagnosis of exclusion defined by the absence of the known causes of lung disease [7]. It is usually characterized by interstitial pneumonia and peripheral bilateral reticulation (thickening of the septae) with honeycombing predominantly peripherally in the lower lobes of the lung, distorted pulmonary architecture resulting in reduced gas exchange and hypoxia, and eventually respiratory failure and death [6]. The only curative treatment available for IPF remains lung transplantation; however, two drugs - nintedinib and pirfenidonehave been approved that slow disease progression although the quality of life has not been positively impacted [8]. Once thought to be a disease caused by chronic inflammation, evidence accumulated over the last decade, including the lack of consistent immune infiltration and the failure of immunesuppression trials (PANTHER, Etanercept etc.) has relegated the notion that inflammation is a major driver of IPF pathogenesis to the sidelines [9]. By the time of diagnosis there are many indications suggesting alterations in immunity, but active inflammation is mostly past its peak $[10,11]$.

A common underlying feature of IPF patients is advanced age. The incidence of IPF is remarkably high in older individuals and a body of evidence has emerged over the past decade highlighting the association of the hallmarks of aging with IPF [12]. These features include dysregulated genomic stability, telomere erosion, mitochondrial dysfunction, senescence, oxidative stress, altered cellular crosstalk, defective nutrient sensing and ER stress, loss of proteostasis (the dynamic regulation of a functional proteome) and defective autophagy [13].

\section{Systemic sclerosis}

Systemic sclerosis is an auto-immune 'orphan' disease characterized by vascular damage and immune activation followed by progressive and unresolved fibrosis of the skin and some internal organs, most commonly the lung. Immune dysregulation and microvascular damage in the skin and internal organs are hallmarks of SSc [14]. SSc presents with enormous heterogeneity, potentially resulting from a complex network of interactions between structural and inflammatory components including different cell types, cytokines and chemokines as well as components of the extra-cellular matrix. SSc can vary widely in terms of disease manifestations and patient outcomes and clinically SSc is a condition with a high unmet medical need [14]. From the physician's perspective, once the diagnosis is made, the parameters used to determine potential disease progression and treatment choices are poorly defined and there is as yet no robust approach to patient stratification using diagnostic methods and clinical biomarkers. Although SSc can be classified into diffuse cutaneous SSc (dcSSc) or limited cutaneous SSc (lcSSc) based on the extent of skin involvement [15], a significant proportion of patients cannot be placed in either category and recent efforts have tried to improve subset classification of SSc using various biomarkers, gene signatures and combinations of clinical parameters. Both $\mathrm{dcSSc}$ and $\mathrm{lcSSc}$ can present with pulmonary involvement, impacting the vasculature as well as the parenchyma of the lung [16]. Interstitial lung disease sometimes represents the initial clinical presentation of SSc [17] while severe ILD manifests in SSc patients within 5 years of initial diagnosis [14]. Denton and Khanna [14] and Distler et al. [18] have extensively reviewed the need for a better understanding of SSc disease heterogeneity to effectively design and execute therapeutic trials. Overall, the prognosis in SSc remains poor, and dcSSc has a 10 -year mortality rate of $\sim 20 \%$.

Many of the genetic associations in SSc are related to inflammation and adaptive immunity and it is not unreasonable to speculate that aberrant immune activation following environmental triggers initiate SSc pathogenesis. The presence of disease-specific auto-antibodies prior to the onset of symptoms or the diagnosis of SSc is consistent with this hypothesis [19] and the aberrant autoimmune response at the onset of SSc appear to be directed towards the endothelial cells in small blood vessels [20]. Many recent studies have investigated the mechanistic details of the initial vascular injury in SSc and its contribution to pathogenesis [21]. Attempts are also being made to "back-translate" findings in the clinic and correlate these findings with mechanistic studies. The injured endothelium appears to undergo defective repair-with dysregulated angiogenesis and aberrant vasculogenesis resulting in the development of the specific structural changes in small vessels that are characteristically seen in SSc [22-24].

\section{Section two: genetic, epigenetic and environmental triggers for the induction of fibrosis}

A wide variety of triggers can result in the development of progressive fibrotic disease either by causing persistent injury or apoptosis of parenchymal cells. These triggers include inherited germline mutations, recurrent or persistent infections, chronic exposure to irritants and particulates like smoke and silica, and immune-mediated chronic inflammation [13]. Regardless of the initiating events, the common underlying feature of all fibrotic diseases is the accumulation of abnormally high numbers of myofibroblasts that are responsible for the excessive deposition of ECM 
(extracellular matrix) components that directly impact organ function [25]. Many fibrotic conditions are accompanied by a robust state of inflammation that is well modulated during tissue homeostasis and physiologic wound repair; any external triggers that disrupt this balance can result in a state of chronic inflammation leading to fibrosis. There are conditions where immune cells may trigger fibrosis as has been described in SSc, asthma, IgG4-related disease and NASH, to cite but a few examples, some of which will be described below. However, in some diseases like IPF, progressive fibrogenesis may occur in the absence of any evidence of an active inflammatory state as revealed by examination of lung tissues from IPF patients [26]. Progressive fibrosis also results from dysregulated interactions between the epithelial and mesenchymal compartments of a tissue or organ, a mechanism that has been proposed for the pathogenesis of IPF [27]. In addition, epithelial-to-mesenchymal transition has been postulated to be a mechanism triggering fibrosis in the context of cancer, as well as in IPF [28]. Although we have categorized triggers into discrete groups, clearly there is overlap between these groups.

\section{Inherited mutations affecting epithelial cell homeostasis}

Many genetic variants that contribute to susceptibility to fibrotic diseases affect the function of the parenchymal cells of the affected organ in a given disease. Some genetic variants affect all cells in the body but may have more prominent effects in certain tissues and organs. IPF is a good example of a disease in which a range of mutations/polymorphic variants alter the function of bronchoalveolar epithelial cells [29]. In general, these mutations either disrupt the barrier (thus making the cells more susceptible to environmental insults) or induce cell-intrinsic changes such as cellular senescence, that contribute to the development of fibrosis.

The dominant risk factor for IPF is a polymorphism in the promoter of the $M U C 5 B$ gene, a gain-of-function mutation that results in excessive production of the MUC5B mucin that is found both in conducting airways as well as in distal airways [30]. Excess MUC5B in lung epithelia is postulated to impair mucociliary clearance in the context of some environmental insults to the bronchoalveolar epithelium and may thus trigger fibrosis specifically in the context of the lung. Desmoplakin (DSP) is a component of desmosomes and genetic variants of the DSP gene that contribute to increased DSP expression in the lung also contribute to IPF susceptibility by presumably causing desmosomal dysfunction and loss of epithelial barrier function [31]. IPF-related variants of $A K A P 13$, that encodes a RhoGEF, also likely contribute to epithelial barrier dysfunction [32].

Variants of genes that encode for surfactant proteins SP-B, SP-C and SP-A1/2 (SFTPB, SFTPC and SFTPA1/2, respectively) are associated with various lung diseases [33]. These protein variants result in protein misfolding, increased ER-stress and a dysfunctional epithelial cell phenotype that facilitates tissue remodeling and fibrosis [34, 35].

Mutations in many genes that contribute to the generation and maintenance of telomeres (TERT, TERC) result in shortened and dysfunctional telomeres which trigger a DNA damage response [36-39]. Rare variants of other genes that contribute to telomere biogenesis and maintenance (DKCl, PARN, RTEL1, TINF2) have been described in familial pulmonary fibrosis [40-43]. The DNA-damage response that is initiated at dysfunctional telomeres can be pro-fibrotic and is linked to a wide spectrum of ILDs and can initiate cell cycle arrest and lead to premature senescence and apoptotic loss $[44,45]$. Aberrant telomere function seems to directly contribute to alveolar stem cell failure, defective alveolar repair and fibrosis possibly because the rate of cell loss exceeds the rate of replacement [46-48]. Variants in telomeraserelated genes are found in about a quarter of all patients with familial IPF, and in about $10 \%$ of patients with sporadic IPF. About half of all the patients with these mutations have a non-IPF diagnosis but comparable rates of progression, lung function decline and survival [49]. Mutations in genes that contribute to telomere function are also mutated in about $10 \%$ of SSc-ILD patients. TERT and TERC mutations have also been linked to liver cirrhosis.

Polymorphic variants in autophagy genes are an important component of Crohn's disease, although these variants have not been specifically linked to patients who present with strictures. In SSc, however, ATG5 is linked to susceptibility suggesting that defective autophagy might contribute to this fibrotic disease. Polymorphic variants in a number of collagen genes that encode subunits of Type IV, Type V, Type XIII and Type XXII collagen have all been linked to the diffuse cutaneous form of SSc as has the CTGF (connective tissue growth factor) gene whose protein product which will be mentioned later in this review.

\section{Mutations in immune-related genes}

When considering human fibrotic diseases, IPF is a good example of a disorder in which the immune system is not considered to be a central player (although inflammation and immune activation are likely very relevant as described below), while SSc is an excellent example of a fibrotic disease in which immune mechanisms are thought to predominate. Many polymorphic variants in genes that affect the function of immune cells are, however, linked to IPF. TGF- $\beta$ has pleomorphic functions that go beyond the immune system and interestingly TGF- $\beta$-related mutations are seen in IPF but not in SSc [50]. Mutations in TLR3, an endosomal TLR that responds to double-stranded RNA and TOLLIP, which encodes a protein that contributes to 
the turnover of the IL-1R and of some Toll-like receptors are also seen in IPF [51]. Polymorphisms in ILIRN that encodes IL-1RA, the IL-1 receptor antagonist, are also relevant in IPF [52, 53], as is a polymorphism in the gene encoding the IL-8 chemokine [54]. These variants suggest an important role for innate immunity in IPF. A likely functional role for adaptive immunity and effector $\mathrm{T}$ cells in IPF is also suggested by the fact that an HLA-DRBI polymorphism is also linked to IPF [55]. CCL18 is an innate chemokine abundant in human lungs and which is relatively poorly studied since it has no murine ortholog. It is believed to be involved in lung homeostasis. A polymorphic variant in CCL18 that increases levels of this chemokine in the blood is linked to a better prognosis in IPF [56].

SSc is perhaps the best example for an autoimmune etiology for fibrosis, though other autoimmune diseases like IgG4-related disease (IgG4-RD) and lupus nephritis should also be considered in such a context. While there are polymorphic variants in a number of genes that encode extracellular matrix proteins as well as in other non-immune proteins are seen in patients with SSc, most of the polymorphic variants is SSc are in immune genes such as HLA class II alleles, the IRF4 gene that encodes a key transcription factor relevant to many activated $\mathrm{T}$ and $\mathrm{B}$ cells, the $I L 12 \mathrm{~A}$ gene that encodes a subunit of the IL-12 cytokine, the $I L-12 R B I$ gene that encodes a subunit of the IL-12 receptor and the STAT4 gene, which is activated downstream of the IL-12 receptor [57].

\section{IPF risk factors: environmental factors, comorbidities and viral infections}

Persistent exposure to multiple environmental factors including dust, fibers, fumes and particulate matter, mostly associated with occupational hazards, air pollution and smoking results in a number of fibrotic lung diseases like IPF, COPD, NSIP and others [58, 59]. In addition, comorbidities like COPD/emphysema, pulmonary hypertension, GERD, diabetes mellitus and obstructive sleep apnea can lead to lung fibrosis. These are discussed in detail elsewhere $[59,60]$ and will be alluded to only in the context of fibrotic triggers for the rest of the discussion here. In addition, viral infections particularly Hepatitis C virus (HCV), EBV infections and other herpes virus infections have been associated with increased risk of pulmonary fibrosis exacerbations and progression of disease [61, 62]. With the emergence of the SARS-COV2 coronavirus pandemic, and the staggering number of cases and the severity of disease in many individuals, there is an urgent need to consider the long-term implications of chronic respiratory symptoms and fibrotic lung disease resulting from these severe infections [63].

\section{Section three: the induction, perpetuation and progression of fibrosis in human disease}

The precise sequence of events that manifest as pathogenic fibrosis are not well established. However, certain risk factors associated with fibrotic diseases like IPF, including genetic predisposition, environmental factors, smoking etc. initiate a complex sequence of altered lung homeostasis that results in progressive fibrosis. In this section we outline the different stages of fibrosis highlighting initiation, perpetuation and progression of pathogenic fibrosis in human lungs.

\section{Damage to epithelial or endothelial cells and induction of pathogenic cellular networks}

The induction of cellular senescence and apoptosis are all likely triggers for fibrosis as implied in the section on non-immune mutations above. Senescent epithelial and endothelial cell states and their depletion resulting from a combination of underlying triggers discussed earlier have been shown to mediate pro-fibrotic pathways. Many of the genetic associations identified from GWAS studies in IPF directly impact epithelial cell homeostasis.

Type-2 alveolar epithelial cells (AEC2s) are the cuboidal surfactant producing cells that maintain homeostasis in alveolar epithelium and its regeneration following injury. AEC2s are self-renewing progenitor cells that differentiate into very large and thin type-1 alveolar epithelial cells (AEC1s) which specialize in gas exchange in the lung [27, 64-67]. During lung homeostasis and repair, $\mathrm{AEC} 2$ is required for alveolar regeneration by differentiating into AEC1 [68, 69]. Many fibrotic diseases of the lung, including IPF and SSc-ILD are associated with dysfunctional or depleted AEC2s [70]. Targeted ablation of AEC2, induction of AEC2 senescence and blocking stemness of AEC2s in mice is sufficient to induce dysfunctional epithelia and lung fibrosis [71-73]. Senescent epithelial cells secrete pro-fibrotic mediators including IL-1 $\beta$, IL-6 and IL-8 which promote fibroblast to myofibroblast differentiation as well as their resistance to apoptosis leading to the accumulation of a fibrotic mass composed of the accumulated myofibroblasts and the extracellular matrix [74-76]. A balance of TGF- $\beta$ and BMP-pathways modulate $\mathrm{AEC} 2$ to $\mathrm{AEC} 1$ differentiation, and this balance is disrupted in fibrotic lung diseases with increased TGF- $\beta$ signaling and abrogation of BMP signaling [77]. In addition, regulated IL-1 $\beta$ levels are required for AEC2 reprogramming during alveolar regeneration but sustained IL- $1 \beta$ availability blocks the generation of mature AEC1 and defective re-epithelization [77].

A recent study used alveolosphere organoid cultures to identify a novel transient cell state between AEC2 and 
AEC1s which was aptly named pre-alveolar type 1 transitional cell state (PATS) [78]. PATS arise from AEC2s following injury and rapidly differentiate into AEC1s. These cells exhibit gene signatures of the DNA-damage response and express senescence-related genes. Cells with features resembling PATS are enriched in the lung in human fibrotic diseases such as IPF and SSc-ILD [72, 78]. Senescent AECs were also recently shown to undergo trans-differentiation into a KRT ${ }^{+}$transitional cell state with epithelial and mesenchymal cell properties during alveolar regeneration following injury [79]. Similar cells that show a KRT5-/KRT17 ${ }^{+}$phenotype accumulate in human lung fibrosis and have been recently termed as 'aberrant basaloid cells' [79, 80]. Alveolar epithelial cells in fibrotic lungs from ARDS show a KRT $8^{+} / \mathrm{KRT} 17^{+}$phenotype and this suggests a potential role for these transitional cell states in multiple fibrotic conditions. These data are consistent with what has been described as epithelialto-mesenchymal transition (EMT) in IPF pathogenesis, in which epithelial cells obtain mesenchymal characteristics, including change in morphology, increased motility and expression of mesenchymal markers like N-Cadherin, Vimentin and a-smooth muscle actin [81, 82]. TGF- $\beta$ has been shown to be a driver of EMT through its effects on SNAI1, SNAI2, TWIST and ID2 [83]. The absolute role of EMT in IPF pathogenesis remains debatable as lineage tracing experiments so far have failed to demonstrate full trans-differentiation of epithelial cells into fibroblasts or myofibroblasts [84]. In the context of many cancers, microRNAs have been shown to play a big role in EMT $[85,86]$ and microRNAs have also been implicated in IPF with reports on down-regulation of let-7d, mir-29 and mir30 as well as upregulation of mir-155 and mir-21 [87, 88]. The loss of epithelial barrier function could trigger lowlevel chronic inflammation that results in the generation of profibrogenic macrophages and is permissive for the activation of fibroblasts. Cytokines generated by myeloid immune cells that activate fibroblasts and myofibroblasts include TGF- $\beta$, FGF, PDGF and Galectin-3 [89].

Oxidative stress has been implicated in IPF pathogenesis $[90,91]$. Two recent articles summarize critical reviews of the multiple IPF studies studying oxidative stress, highlighting the observations that strongly implicate oxidative stress as one of the major factors contributing to IPF pathogenesis [91, 92]. Oxidative stress in lungs results from environmental factors such as cigarette smoking induced ER stress and ROS production [93], and production of NADPH oxidase (NOX4) by immune as well as lung structural cells in response to TGF- $\beta$ stimulation [94]. TGF- $\beta$ appears to drive mitochondrial ROS (mtROS) production associated with pro-fibrotic reprogramming of lung cells [94]. NOX4 activation has been reported to suppress both mitochondrial biogenesis and bioenergetics in lung fibroblasts, while
NOX4's pharmacological inhibition, or its genetic silencing, has been shown to restore them [95]. Increased oxidative stress appears to induce premature senescence of the cells as a result of which fibroblasts acquire apoptosis resistance and persist to stay metabolically active producing high levels of reactive oxygen species (ROS) [96-98]. One of the major components of the oxidative stress pathway is STAT3 activation [99] which has been shown to make fibroblasts resistant to apoptosis [100]. More recently, thyroid hormonemediated reduction in STAT3 signaling was shown to significantly resolve lung fibrosis, further supporting a role for STAT3 activation in persistent fibroblast activation and fibrosis [101]. Details of the mechanism of fibrosis driven by oxidative stress, ER stress, hypoxia and senescence-mediated altered lung homeostasis are provided elsewhere [13].

SSc may be initiated by endothelial cell injury and presents as a vasculopathy [102]. The exact mechanism by which endothelial cells are injured is unclear, and how exactly endothelial cell injury results in fibrosis is also not clear. Vascular endothelial cells under oxidative stress appear to exhibit cell-fate plasticity and undergo endothelial-to-mesenchymal transition (EndoMT) into cells with myofibroblast like features, thereby causing vascular damage [102, 103]. Additionally, increased ROS generation appears to mediate TGF- $\beta$-induced EndMT in SSc and several other conditions including atherosclerosis and diabetic neuropathy [104-106]. Given its profibrotic role, TGF- $\beta$ mediates EndMT through both Smad-dependent and Smadindependent pathways, with the involvement of numerous transcriptional regulators such as Snail1, Snail2, Twist and some Zeb family members [107-110]. In addition, Endothelial cell derived endothelin-1, which is highly upregulated in SSc-skin and SSc-ILD lung appears to potentiate TGF$\beta$-mediated EndMT [111]. A detailed review on the links between oxidative and EndMT can be found elsewhere [112].

Caveolin-1 (CAV1) expression is down-regulated in affected tissues from SSc and SSc-ILD as well as IPF lungs [113] and restoration of CAV1 functional domains using synthesized peptides and adenoviral-expression reversed phenotypes of SSc and IPF in vitro and in vivo in animal models of PF $[114,115]$. Notch and $W n t / \beta$-catenin pathways also synergize to mediate EndMT in vitro and in mouse models of fibrosis [116, 117]. These studies have demonstrated that EndMT is not a phenomenon restricted to experimental animal models since it was observed in studies performed on lung tissues from patients with SSc-ILD and PAH (pulmonary arterial hypertension). Given the pleotropic effect of many of these pathways, further molecular understanding of this process is, however, needed to ensure safe therapeutic strategies for SSc-related fibrosis.

We have described an immune-mediated mechanism for endothelial cell apoptosis in SSc (discussed below) but have 
not obtained knowledge about the downstream events that could lead to fibrosis. It is possible that injured endothelial cells release pro-fibrogenic proteins in a manner similar to that postulated for injured epithelial cells. Whether endothelial damage is responsible for some degree of hypoxia and consequent induction of fibrosis is also unclear.

\section{Immune-mediated events leading to fibrosis}

Epithelial cell damage frequently occurs in the context of viral infection and in genetically susceptible individuals this can lead to fibrosis. Well-established examples are chronic infections with the hepatitis $\mathrm{B}$ and the hepatitis $\mathrm{C}$ viruses that are the major causes of liver cirrhosis when the disease is viewed in a global context. Progression from hepatitis to cirrhosis can take a decade or two, and the chronic inflammatory process that results from the activation and reactivation of virus-specific $\mathrm{CD}^{+}$helper $\mathrm{T}$ cells that secrete inflammatory cytokines and the ongoing albeit somewhat inefficient elimination of infected liver cells by cytotoxic $\mathrm{CD}^{+} \mathrm{T}$ cells, both contribute to the fibrotic process. The overall adaptive immune response in this context is biased towards a Type 1 response including NK cells, TH1 cells and $\mathrm{CD} 8^{+}$CTLs, though the latter are likely in a state of partial exhaustion.

\section{Innate immune pathways facilitating fibrosis}

Inflammation driven by innate and adaptive immune responses are a common feature in many fibrotic diseases, including IPF, where immune infiltrates tend to be abundant in monocytes, plasmacytoid dendritic cells, mast cells and neutrophils. The inflammation is heterogeneous across most of the organs and varies among different diseases, but accumulation of activated macrophages and monocytes appears to be a common feature of most fibrotic diseases. However, the extraordinary plasticity and pleiotropy of macrophages and monocytes has made establishing the pathogenic vs homeostatic roles of these cells a challenge and there are no successful macrophage/monocyte targeting therapies currently available. Macrophages/ monocytes respond to a number of stimuli including PAMPs, DAMPs, apoptotic cell debris, cytokines and chemokines. TLR4 signaling appears to be a key mediator of fibrosis and blockade of TLR4 signaling leads to reduction and reversal of fibrosis in several preclinical disease models [118]. The ligands for TLR4 in SSc, IPF and other fibrotic diseases have been shown to be the alternatively spliced forms of two matri-cellular proteins tenacin-C and fibronectin-EDA, HMGB1, S100 and hyaluronan fragments [119-123]. Other TLRs implicated in SSc are TLR7, TLR8 and TLR9 which seem to be at least partly modulated by TGF $\beta$ production $[124,125]$. Chronic activation of plasmacytoid DCs (pDCs) through TLR8 and TLR9 may mediate SSc pathogenesis by induction of IFN $\alpha$ and CXCL4 [124]. Proinflammatory cytokines IL-1 and IL-6 are also produced downstream of TLR signaling and have been established to be key drivers of fibrosis in SSc; they have also been implicated in IPF pathogenesis [126-128]. Blockade of the IL6R, however, resulted in an increase in serious infections in treated patients and resulted in the termination of a clinical trial [129]. IL-6 also seems to act downstream of or with lysophosphatidic acid (LPA) to induce an autotaxin dependent self-perpetuating loop of fibrosis in experimental models of skin fibrosis [130], and levels of all these three mediators are elevated in SSc skin.

M2-like and monocyte-derived macrophages also have key roles in lung, liver and skin fibrosis. M2-polarized macrophages differentiate in the presence of the two major type 2 inflammatory cytokines, IL-4 and IL-13. Since there is an ongoing debate about the validity of the rigid classification of macrophages into the M1 and M2 subtypes, especially when most tissue studies use a single marker, we prefer to describe these cells as M2-like. M2-like polarized macrophages are abundant in fibrotic tissues including lung and skin from SSc patients, and SPP $1^{+}$macrophages which resemble M2-polarized macrophages in many ways are found in the lungs of IPF patients [131]. M2-like polarized macrophages secrete profibrotic growth factors like TGF- $\beta$, FGF, PDGF $\alpha$, IGF1 and VEGF [132]. Most of the inferences thus far about role of type 2 inflammation in mediating lung and skin fibrosis are derived from extrapolating findings from murine models like bleomycin induced lung and skin fibrosis or they are conclusions drawn from human studies that show an association with pathogenic type 2 responses like increased periostin and buildup of IL-4- and IL-13-related pathways (Fig. 2). However, a recent study involving the therapeutic blockade of two of the key cytokines of type 2 immunity, IL-4 \& IL-13, underscores the importance of type 2 inflammation in dermal fibrosis [133]. This study establishes one of the first direct lines of evidence to connect type 2 inflammation in human fibrosis. These therapies lead to disease modification by blocking the cellular network of type 2 innate and adaptive immune responses and more studies are warranted to gain further understanding of fibrosis linked to type 2 inflammation.

\section{Adaptive immune pathways facilitating fibrosis}

The abundance of immune infiltrates composed of specific subsets of T cells and B cells and the presence of autoantibodies in diseases like SSc underscores the importance of adaptive immunity in fibrosis [134]. The T cell infiltrate in fibrotic diseases tends to be very heterogeneous and a number of $T$ helper subsets have been described to have profibrotic roles, though many of the inferences and models are derived from experimental preclinical mouse models. 
Fig. 2 Type 2 immunity and the end-stage events in fibrosis

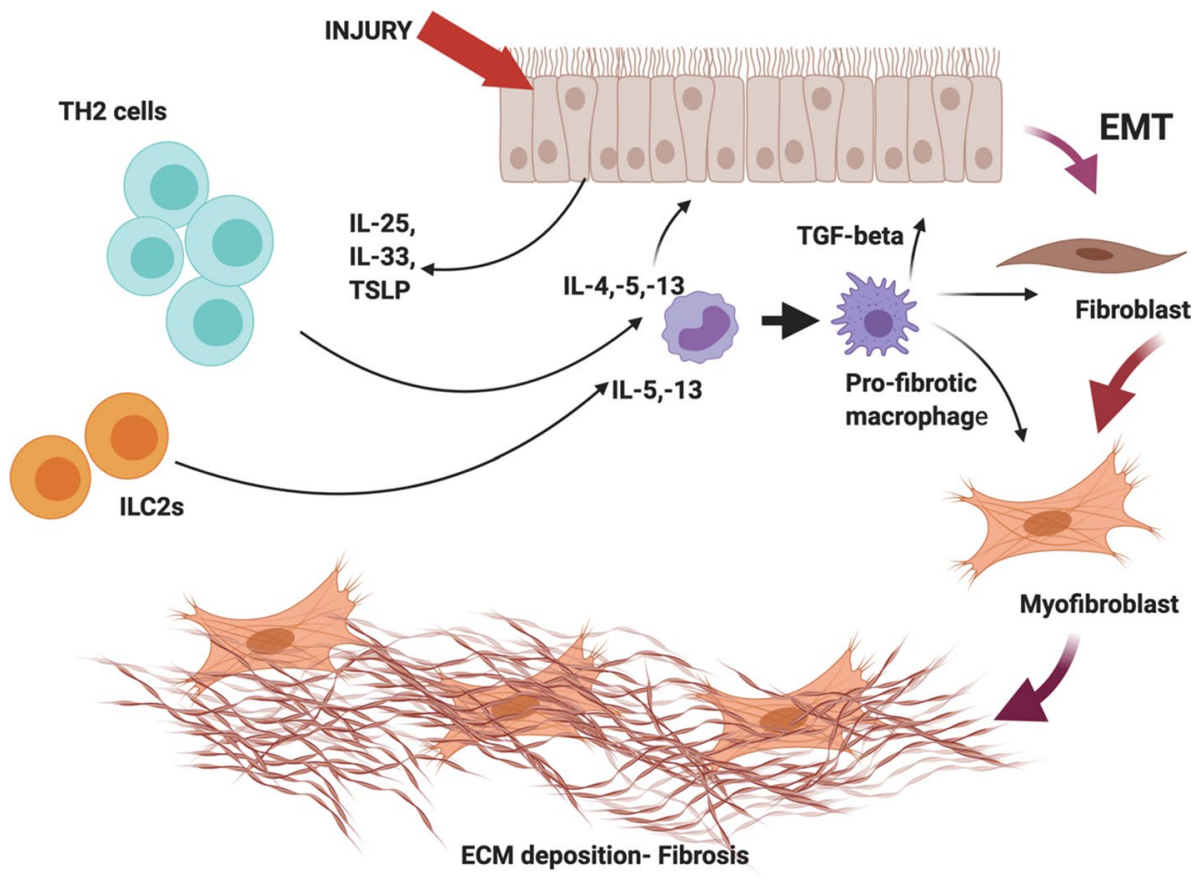

The ideal scenario of establishing a direct role of a cell type in fibrosis is to demonstrate its abundance in fibrotic tissue and to establish that pathology is attenuated when the cell type is depleted. The latter is obviously hard to achieve in humans, except in the settings of clinical trials; however, many therapeutics tend to have a broad effect that does not typically reveal a specific disease mechanism. One example is that the role of $\mathrm{T}$ cells can only be very broadly inferred in SSc pathogenesis since immunosuppressive modalities like cyclophosphamide (CYC) remain the main therapeutic option, especially in SSc-ILD despite its caveats [135, 136]. A number of studies have made the case for the role of T-helper type-2 (Th2)-oriented immune response with key roles for interleukin (IL)-4 and IL-13; however, a contribution of Th2 cells has never been directly established, and the perceived Th2 bias could reflect the contribution of innate immune cells that secrete IL-13 as discussed above.

We have identified abundant infiltrates of cytotoxic $\mathrm{CD}^{+}$ T cells in tissue biopsies from IgG4-RD, SSc and fibrosing mediastinitis (a fibrotic disease linked to Histoplasma capsulatum infection)[137-140]. Quantitative examination of all major T cell subsets in early diffuse SSc, revealed the clonal expansion of $\mathrm{CD}^{+}$CTLs in the blood and the presence of $\mathrm{CD} 4^{+}$CTLs in skin biopsies, outnumbering all other $\mathrm{CD} 4^{+}$ $\mathrm{T}$ cell subsets in the tissues of most patients. Th2 cells were not abundant when $\mathrm{CD}^{+}{ }^{+} \mathrm{T}$ cell subsets were quantitated in the tissues. Both in IgG4-RD and SSc-skin CD ${ }^{+}$CTLs may be responsible for inducing apoptotic death of cells in disease tissues, [138, 139]. The majority of the apoptotic cells in SSc tissues were endothelial cells. CD4 ${ }^{+}$CTLs may also be a source of IL- $1 \beta$ as well as TGF- $\beta$ and these cytokines may also contribute to profibrotic events along with the innate immune sources [87, 88] (Fig. 3). Activated and apoptotic endothelial cells show increased ICAM-1 and GlyCAM-1 expression leading to accumulation of Th2/Th17 cells, macrophages and mast cells that promote inflammation and aggressive tissue remodeling. More recently, $\mathrm{T}$ follicular helper cells have been shown to be present in SSc skin, though the studies were not quantitative; these cells might contribute to dermal fibrosis in an IL-21 and Mmp12 dependent manner [141, 142].

The depletion of $\mathrm{B}$ cells results is dramatic improvement in IgG4-RD and there are some data that argue that B cell depletion may benefit patients with SSc as well. Since there is no firm evidence to suggest that autoantibodies contribute to fibrosis in either of these diseases, we posit that activated $\mathrm{B}$ cells in lesions may be involved in antigen presentation to $\mathrm{CD}^{+} \mathrm{T}$ cells or that they perhaps secrete pro-fibrotic molecules. While in IgG4-RD, a much less severe fibrotic disease than SSc, reversal of fibrosis has been observed with anti-CD20-mediated B cell depletion, the exact mechanisms by which $\mathrm{B}$ cells contribute to fibrosis remain to be established.

\section{Mechanisms of perpetuation and progression of fibrosis}

The cell intrinsic changes in epithelial and endothelial cells resulting from senescence, genetic predisposition or environmental stress and their dysregulated crosstalk, along with the ensuing innate and adaptive immune responses create 
Fig. 3 Cytotoxic T cells and the induction of fibrosis

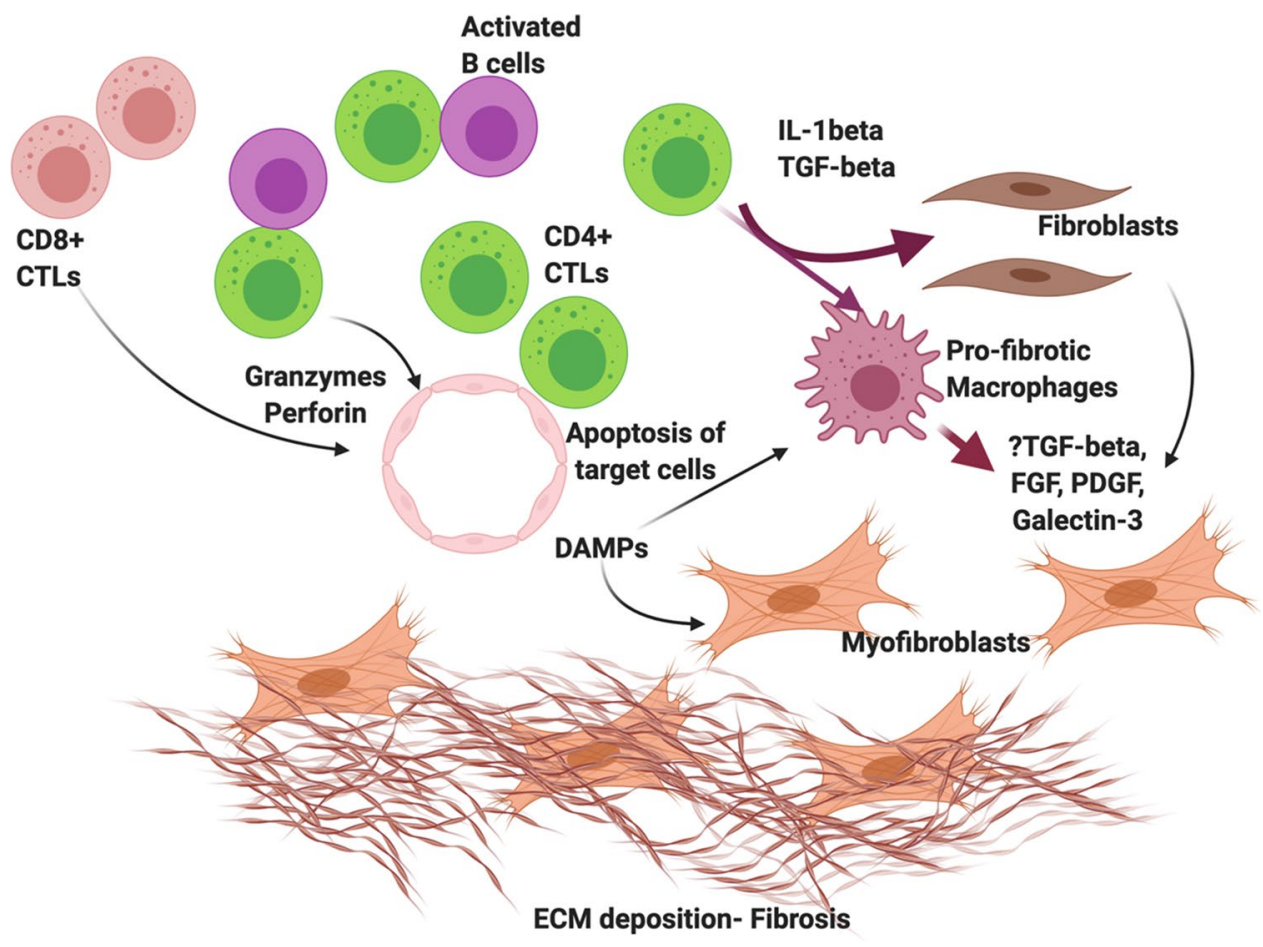

cellular networks that contribute to perpetuation and progression of fibrosis in diseased tissues.

The active form of TGF- $\beta$ is abundant in bronchoalveolar lavage collections from IPF patients and is over-expressed in IPF and SSc-derived fibroblasts. TGF- $\beta$ mediates an increase in connective tissue synthesis by inducing fibroblast activation, myofibroblast differentiation, secretion of ECM proteins and increased expression of inhibitors of connective tissue proteases. TGF- $\beta$ is expressed in dysfunctional epithelial cells, activated fibroblasts as well as the infiltrating macrophages in IPF tissue and it induces the increased expression of a number of pro-fibrotic growth factors and cytokines like CTGF and IGF1. CTGF has emerged as an important mediator of fibrosis downstream of TGF- $\beta$ and is emerging as one of the most promising therapeutic targets in treating IPF, as will be discussed later in this review. Other important mediators of fibroblast activation and persistent tissue remodeling include TNF- $\alpha$, PGE2, MCP-1, FGF-2, PDGF and others and these are described in detail elsewhere [13, 143]. These cytokine/growth factor-driven pathways result in an increase in connective tissue mass and increased stiffness of the affected organs.

The cellular origins of myofibroblasts in lung, skin, liver and other organs have been assessed using genetic tools for cell-fate tracing [144-147]. Given their contribution to the self-perpetuating, progressive phase of fibrosis, identifying the main cellular source of myofibroblasts is important to understand the pathogenesis of fibrotic diseases and to identify druggable pathways and cell states. Myofibroblasts have been demonstrated to originate from resident fibroblast subsets, mesothelial cells, pericytes and potentially from epithelial and endothelial compartments following their transition to mesenchymal-like cell states. A detailed review on the origins of myofibroblasts in fibrotic disease can be found elsewhere [148].

In many disease settings, fibrogenesis reaches a tipping point where fibroblast activation, proliferation and altered extracellular matrix (ECM) deposition becomes a self-amplifying loop independent of any of the mechanisms of initiation/perpetuation discussed thus far. The ECM is a source of critical spatial and contextual cues under homeostatic conditions, and its altered composition and stiffness in fibrotic diseases drive many pathways of fibroblast proliferation, migration, and production of pro-fibrotic mediators. The mechanisms of end-stage progressive fibrosis involve a set of cell-cell and cell-ECM interactions that add another layer of complex biology to fibrosis in human disease. Recent studies have focused on establishing the ECM-mediated and mechano-sensory driven feed-forward pathways that mediate a self-amplifying loop of progressive fibrosis $[149,150]$. The composition as well as mechanics of the ECM profoundly influence the biology of tissue fibrosis and are among the most pursued fields of investigation in drug discovery efforts towards fibrotic diseases. Fragments of ECM components influence myofibroblast differentiation by modulating the activity of many pro-fibrotic stimuli including TGF- $\beta$ and integrin signaling and the mechanosensitive Hippo pathway effector Yes-associated protein 1 (YAP1). Active TGF- $\beta$, 
integrin and YAP signaling and sustained increase of miR21 may drive progressive fibrosis in the absence of ongoing injury, creating fibrogenic niches and the formation of fibroblastic foci leading to progressive fibrosis [25]. Given its role in the progression of fibrosis, a comprehensive understanding of the ECM in homeostasis and disease is essential to fully capture the mechanisms of controlled tissue repair and progressive fibrosis. New evidence is emerging regarding the alterations in ECM composition in the context of the aging lung both at the transcriptome and proteome levels that could shed some light on the mediators of the alteration of the properties of the ECM [151]. The composition of the ECM in IPF and COPD lung for example is considerably different from that in normal lungs and the ECM has been a source of target identification for novel therapeutic approaches in fibrotic lung diseases [152, 153].

The failed resolution and altered ECM in fibrotic diseases like IPF results in increased tissue stiffness that is functionally implicated in fibroblast activation and migration, another major player in the feed-forward loop of progressive fibrotic tissue remodeling. Mechanosensory components like cell adhesion protein complexes (including many integrins) and ligand gated ion channels exhibit distinct features in fibrotic scars and contribute to persistent altered endothelial and epithelial cell states as well as activated fibroblast/ myofibroblast states, as discussed extensively by Tschumperlin et al. [154]. Lessons gleaned from these altered states in disease vs homeostasis warrant further investigation and may be translatable into novel clinical and therapeutic interventions that target fibrotic tissue remodeling $[154,155]$.

\section{Section four: lessons from systems-based approaches to investigate mechanisms of pathogenic fibrosis}

Fibrotic diseases have been historically difficult to study and the approaches towards obtaining a mechanistic understanding of disease pathogenesis have evolved over time. In the past fibrosis research focused on experimental models, mostly the bleomycin model in parallel with in vitro studies on fibroblasts under different conditions. These models for the most part do not resemble most relevant human fibrotic diseases. More recently, a number of animal models have been engineered that replicate different pathogenic features described in IPF/SSc/NASH and are more suitable for discovery and pre-clinical testing of candidate therapeutic drugs [156]. Over the years, human biopsies and tissue samples have been studied using newer modalities and systematic efforts towards creating consortia and tissue banks have resulted in some well-planned studies. In addition, advances in high throughput data-generation including genomics, proteomics, metabolomics and studies on the microbiome have significantly advanced our understanding of a number of fibrotic diseases of the lung, liver and skin [157].

Several large efforts have been in place to understand the molecular features of IPF lungs with the goal of capturing the heterogeneity of disease pathology and altered cellstates and cell types. Genome wide transcriptomic studies of human lung tissues in IPF have led to the identification of novel pathways and molecular targets using systemsbiology methods as well as more reductionist experimental approaches [158]. Distinct gene-expression patterns have been effectively used to identify potential aberrant developmental pathways in IPF lungs [159], have contributed to the discovery of defective mitochondrial homeostasis and function $[101,160]$ and to the recognition of different patient phenotypes and disease subsets $[161,162]$ and many other predictions as well.

The transcriptomic studies mentioned above have advanced our understanding of fibrotic diseases in terms of potential mechanistic pathways and biomarker profiles of disease subsets as well as drug responses. However, these studies even with the best mechanistic deconvolution are limited by changes in the cellular composition of fibrotic tissues and may not necessarily be identifying altered 'pathogenic cell-states'. The advances in single cell technology over the last 5 years has made it possible to identify novel previously un-identified types and cell states that have helped provide more nuanced insights into the pathophysiology of human diseases [163, 164]. The human cell atlas project is a great example of how a concerted effort has led to a wealth of comprehensive information about molecular cellstates in 'healthy' and 'diseased' human tissues [165]. In the last 3 years, a number of single cell RNA-Seq studies have helped make significant inroads into the multi-cellular complexity of fibrotic tissues and the understanding of complex cellular networks that may drive the pathogenic processes involved in fibrosis. Single cell profiling of epithelial cells sorted from fibrotic lungs of IPF patients identified three different subsets-one with basal cell characteristics, one with features of goblet cells from conducting airways and an atypical transitional cell state that co-expressed AEC2-associated cell signatures [70]. A novel population of CCR $10^{+}$EphA3 $3^{+}$ epithelial cells was identified from IPF lungs that showed transcriptomic signatures of altered mitochondrial function and enrichment of pro-fibrotic pathways [148]. These epithelial cells promoted lung fibroblast and COL1A1 secretion and also induce lung remodeling in humanized NSG mice. A comprehensive study published recently took a deeper dive into the characterization of cell types and cell states by studying fibrotic lungs from 32 IPF and 18 COPD patients with a total of 312,928 cells identifying 38 distinct cell types [80]. This dataset is readily accessible to the public on the IPF cell atlas and identifies a novel 'aberrant basaloid' cell type that co-expresses basal epithelial, mesenchymal, senescence 
and developmental markers, lines fibroblastic foci and potentially activates TGF- $\beta 1$-dependent and independent profibrotic pathways locally. These cells were also validated from an orthogonal dataset [131]. These studies confirm the aberrant phenotypic and genomic changes seen in IPF lung epithelium. It is, however, not very clear whether these altered cell states result from abnormal de-differentiation of alveolar epithelial cells or from migrating airway epithelial cells that have been altered by the profibrotic micro-environment in the lung interstitium. It is also not clear if there is any overlap between these altered cell states described in IPF lungs. Additional studies with proper 'normal' controls may help resolve some of these outstanding questions.

In addition to the characterization of altered epithelial cells, single cell RNA-Seq has also helped with the identification of disease-related macrophage heterogeneity in fibrotic lungs [131, 166].

\section{Section five: possible future interventions including disease-modifying strategies}

Though there have been some advances, IPF and SSc remain diseases that lack therapies that are highly effective, and the optimal choice or combination of therapeutics is still the subject of debate. Nintedanib can ameliorate a decline in forced vital capacity (FVC) in patients with SSc and lung fibrosis [167], and autologous stem cell transplantation can favorably modify disease course in some patients, leading to remission or even "cure" [168]. This latter finding speaks to the relevance of an underlying causal immune mechanism in this disease.

Well-defined recommendations for clinical management and standardization of diagnostic criteria for IPF patients has made multi-center, randomized placebo-controlled clinical trials possible for potential disease-modifying drugs. One of the biggest shocks to the field were the failed and possibly harmful PANTHER-IPF (Prednisone, Azathioprine and $N$-acetylcysteine triple combo) [9] and warfarin trials [169]. These trials drew the whole field back to the drawing board. Eventually through additional randomized clinical trials, two effective disease-modifying therapies were identified for IPF-nintedanib and pirfenidone [170-172]. Both these drugs are approved for IPF and are fairly effective in slowing the decline in lung function, although they both have serious side effects. More recently, nintedanib has proven beneficial for improved lung function in SSc-associated lung disease [167]. Both these compounds, however, slow the progression of these diseases and there is a need for alternative therapeutic approaches to not just slow disease progression but to also reverse it. Recent studies have encompassed increased efforts to enhance contextual regenerative pathways in the lung and liver with a focus on restoring a functional epithelium.

In the last decade, a number of biologics have been tested in both IPF and SSc with mostly underwhelming results in clinical settings. Some examples of failed trials in IPF include monoclonal antibodies against CCL2 (Carlumab), LOXL2 (Simtuzumab), TNF- $\alpha$ (Etanercept), IL-13 (Tralokinumab \& Lebrikizumab) and others in which patients showed no noticeable improvement [173-176]. Combination of lebrikizumab and pirfenidone did not meet the primary endpoint of lung function decline.

Not all the news has been bad. Pamrevlumab (FG-3019) a monoclonal antibody against CTGF showed a very promising safety and efficacy profile in a phase 2 randomized, placebo-controlled trial. Blocking CTGF resulted in decreased progression of IPF over a 1-year period with multiple positive efficacy outcomes including lung function and quality of life [177]. Over a period of 48 weeks, $10 \%$ of patients in the Pamrevlumab group experienced disease progression, compared to $31.4 \%$ of patients in the placebo group. Progression of lung fibrosis was also reduced significantly in patients treated with Pamrevlumab [177]. Two novel drugs that also hold potential in improving the outcome of mild-to-moderate IPF patients are the Autotaxin inhibitor GLPG1960 and recombinant human Pentraxin 2 protein PRM-151-202. A Phase 2 trial of GLPG1960 on mild-tomoderate IPF patients showed significantly improved lung function at 12 weeks compared to a lung function decline in the placebo group [178]. This drug is undergoing two phase 3 clinical trials-ISABELA 1 and ISABELA 2 with a total of around 1500 IPF patients. More recently PRM-151-202 has been shown to block the differentiation of proinflammatory macrophages and production of TGF- $\beta 1[179,180]$. A phase 2 double-blinded randomized trial of PRM-151-202 showed significant reduction in rate of decline in FVC and stable 6-min walking distance from baseline compared to placebo at 28 weeks [181]. Long-term assessment of PRM151 also showed good safety and tolerability profiles [182] and a phase 3 clinical trial is ongoing.

For systemic sclerosis, immunosuppressive treatments like cyclophosphamide (CYC) and mycophenolate mofetil (MMF) remain the main therapeutic option, especially in SSc-ILD despite its caveats [135, 136, 183]. Given the abundance of auto-antibodies and the presence of B cell infiltrates in skin samples of LcSSc and dcSSc patients, Rituximab (anti-CD20), which depletes B cells, has been recently tested especially in CYC refractory patients, with some success [184, 185]. A study comparing rituximab on top of standard treatment with standard treatment alone showed a significant and persistent benefit to rituximab treated patients [186]. Based on these initial findings, large scale and properly controlled multi-center trials are needed to establish the benefits of B cell depletion treatments in patients with SSc-ILD. A 
pilot trial on Belimumab (which blocks BAFF) in patients with early dcSSc with background MMF therapy showed promising results with improved MRSS scores and gene expression changes consistent with decrease in expression of B cell signaling with a significant decrease in profibrotic genes and pathways [187]. Recently In addition, a monoclonal bispecific IL-4 and IL-13 antibody which failed in IPF showed a very promising disease modifying trend in mRSS scores in dSSc and robust blockade of biomarkers of type 2 inflammation-like chemokine (C-C motif) ligand 17 [133].

\section{Conclusions}

Although progress has been made both in terms of our understanding of the pathogenesis of fibrosis and in the treatment of fibrotic diseases, our understanding still remains rudimentary, and all current treatments have failed to make a significant dent in the associated morbidity and mortality in diseases like idiopathic pulmonary fibrosis or systemic sclerosis. Fibrotic human diseases and the development of therapies for these diseases therefore continues to pose great challenges for the field.

Acknowledgements We acknowledge many discussions over the years with colleagues.

Author contributions Both authors contributed to the writing and the figures.

Funding SP's support for this area of research comes from NIH U19 AI110495

\section{Declarations}

Conflict of interest HM is an employee of Sanofi; SP is on the SAB of BeBio and Abpro.

\section{References}

1. Distler JHW et al (2019) Shared and distinct mechanisms of fibrosis. Nat Rev Rheumatol 15(12):705-730

2. Helling BA, Yang IV (2015) Epigenetics in lung fibrosis: from pathobiology to treatment perspective. Curr Opin Pulm Med 21(5):454-462

3. Herzog EL et al (2014) Review: interstitial lung disease associated with systemic sclerosis and idiopathic pulmonary fibrosis: how similar and distinct? Arthritis Rheumatol 66(8):1967-1978

4. Solomon JJ et al (2013) Scleroderma lung disease. Eur Respir Rev 22(127):6-19

5. Gabrielli A, Avvedimento EV, Krieg T (2009) Scleroderma. N Engl J Med 360(19):1989-2003

6. Raghu G et al (2011) An official ATS/ERS/JRS/ALAT statement: idiopathic pulmonary fibrosis: evidence-based guidelines for diagnosis and management. Am J Respir Crit Care Med 183(6):788-824
7. Martinez FJ et al (2017) Idiopathic pulmonary fibrosis. Nat Rev Dis Primers 3:17074

8. Somogyi V et al (2019) The therapy of idiopathic pulmonary fibrosis: what is next? Eur Respir Rev 28(153):190021

9. Idiopathic Pulmonary Fibrosis Clinical Research (2012) Prednisone, azathioprine, and $\mathrm{N}$-acetylcysteine for pulmonary fibrosis. N Engl J Med 366(21):1968-1977

10. Gilani SR et al (2010) CD28 down-regulation on circulating CD4 $\mathrm{T}$ cells is associated with poor prognoses of patients with idiopathic pulmonary fibrosis. PLOS ONE 5(1):e8959

11. Ley B, Collard HR, King TE Jr (2011) Clinical course and prediction of survival in idiopathic pulmonary fibrosis. Am J Respir Crit Care Med 183(4):431-440

12. Pardo A, Selman M (2016) Lung fibroblasts, aging, and idiopathic pulmonary fibrosis. Ann Am Thorac Soc 13(Suppl 5):S417-S421

13. Phan THG et al (2020) Emerging cellular and molecular determinants of idiopathic pulmonary fibrosis. Cell Mol Life Sci 78:2031

14. Denton CP, Khanna D (2017) Systemic sclerosis. Lancet 390(10103):1685-1699

15. Wirz EG et al (2016) Incidence and predictors of cutaneous manifestations during the early course of systemic sclerosis: a 10-year longitudinal study from the EUSTAR database. Ann Rheum Dis 75(7):1285-1292

16. Hoffmann-Vold AM et al (2019) Tracking impact of interstitial lung disease in systemic sclerosis in a complete nationwide cohort. Am J Respir Crit Care Med 200(10):1258-1266

17. Rubio-Rivas $\mathrm{M}$ et al (2018) First clinical symptom as a prognostic factor in systemic sclerosis: results of a retrospective nationwide cohort study. Clin Rheumatol 37(4):999-1009

18. Distler O et al (2020) Predictors of progression in systemic sclerosis patients with interstitial lung disease. Eur Respir J 55(5):1902026

19. Burbelo PD et al (2019) Autoantibodies are present before the clinical diagnosis of systemic sclerosis. PLOS ONE 14(3): 0214202

20. Avouac J et al (2011) Preliminary criteria for the very early diagnosis of systemic sclerosis: results of a Delphi Consensus Study from EULAR Scleroderma Trials and Research Group. Ann Rheum Dis 70(3):476-481

21. Pattanaik D et al (2015) Pathogenesis of systemic sclerosis. Front Immunol 6:272

22. Asano $Y$ et al (2010) Endothelial Fli1 deficiency impairs vascular homeostasis: a role in scleroderma vasculopathy. Am J Pathol 176(4):1983-1998

23. Kuwana M, Okazaki Y (2014) Brief report: impaired in vivo neovascularization capacity of endothelial progenitor cells in patients with systemic sclerosis. Arthritis Rheumatol 66(5):1300-1305

24. Yamaguchi $\mathrm{Y}$ et al (2010) Enhanced angiogenic potency of monocytic endothelial progenitor cells in patients with systemic sclerosis. Arthritis Res Ther 12(6):R205

25. Herrera J, Henke CA, Bitterman PB (2018) Extracellular matrix as a driver of progressive fibrosis. J Clin Invest 128(1):45-53

26. Travis WD et al (2013) An official American thoracic society/ European respiratory society statement: update of the international multidisciplinary classification of the idiopathic interstitial pneumonias. Am J Respir Crit Care Med 188(6):733-748

27. Zepp JA et al (2017) Distinct mesenchymal lineages and niches promote epithelial self-renewal and myofibrogenesis in the lung. Cell 170(6):1134-1148

28. Di Gregorio J et al (2020) The epithelial-to-mesenchymal transition as a possible therapeutic target in fibrotic disorders. Front Cell Dev Biol 8:607483

29. Kaur A, Mathai SK, Schwartz DA (2017) Genetics in idiopathic pulmonary fibrosis pathogenesis, prognosis, and treatment. Front Med (Lausanne) 4:154 
30. Seibold MA et al (2011) A common MUC5B promoter polymorphism and pulmonary fibrosis. N Engl J Med 364(16):1503-1512

31. Mathai SK et al (2016) Desmoplakin variants are associated with idiopathic pulmonary fibrosis. Am J Respir Crit Care Med 193(10):1151-1160

32. Allen RJ et al (2017) Genetic variants associated with susceptibility to idiopathic pulmonary fibrosis in people of European ancestry: a genome-wide association study. Lancet Respir Med 5(11):869-880

33. Nogee LM et al (2001) A mutation in the surfactant protein C gene associated with familial interstitial lung disease. $\mathrm{N}$ Engl $\mathbf{J}$ Med 344(8):573-579

34. Tanjore H, Blackwell TS, Lawson WE (2012) Emerging evidence for endoplasmic reticulum stress in the pathogenesis of idiopathic pulmonary fibrosis. Am J Physiol Lung Cell Mol Physiol 302(8):L721-L729

35. Lawson WE et al (2011) Endoplasmic reticulum stress enhances fibrotic remodeling in the lungs. Proc Natl Acad Sci USA 108(26):10562-10567

36. Armanios MY et al (2007) Telomerase mutations in families with idiopathic pulmonary fibrosis. N Engl J Med 356(13):1317-1326

37. Tsakiri KD et al (2007) Adult-onset pulmonary fibrosis caused by mutations in telomerase. Proc Natl Acad Sci USA 104(18):7552-7557

38. di Fagagna FA et al (2003) A DNA damage checkpoint response in telomere-initiated senescence. Nature 426(6963):194-198

39. Hemann MT et al (2001) The shortest telomere, not average telomere length, is critical for cell viability and chromosome stability. Cell 107(1):67-77

40. Kropski JA et al (2014) A novel dyskerin (DKC1) mutation is associated with familial interstitial pneumonia. Chest 146(1):e1-e7

41. Moon DH et al (2015) Poly(A)-specific ribonuclease (PARN) mediates 3'-end maturation of the telomerase RNA component. Nat Genet 47(12): 1482-1488

42. Cogan JD et al (2015) Rare variants in RTEL1 are associated with familial interstitial pneumonia. Am J Respir Crit Care Med 191(6):646-655

43. Alder JK et al (2015) Exome sequencing identifies mutant TINF2 in a family with pulmonary fibrosis. Chest 147(5):1361-1368

44. Takai H, Smogorzewska A, de Lange T (2003) DNA damage foci at dysfunctional telomeres. Curr Biol 13(17):1549-1556

45. Hewitt $\mathrm{G}$ et al (2012) Telomeres are favoured targets of a persistent DNA damage response in ageing and stress-induced senescence. Nat Commun 3:708

46. Alder JK et al (2015) Telomere dysfunction causes alveolar stem cell failure. Proc Natl Acad Sci USA 112(16):5099-5104

47. Naikawadi RP et al (2016) Telomere dysfunction in alveolar epithelial cells causes lung remodeling and fibrosis. JCI Insight 1(14):e86704

48. Povedano JM et al (2018) Therapeutic effects of telomerase in mice with pulmonary fibrosis induced by damage to the lungs and short telomeres. Elife. https://doi.org/10.7554/eLife.31299

49. Newton CA et al (2016) Telomere-related lung fibrosis is diagnostically heterogeneous but uniformly progressive. Eur Respir J 48(6): 1710-1720

50. Xaubet A et al (2003) Transforming growth factor-beta1 gene polymorphisms are associated with disease progression in idiopathic pulmonary fibrosis. Am J Respir Crit Care Med 168(4):431-435

51. O'Dwyer DN et al (2013) The Toll-like receptor 3 L412F polymorphism and disease progression in idiopathic pulmonary fibrosis. Am J Respir Crit Care Med 188(12):1442-1450

52. Korthagen NM et al (2012) IL1RN genetic variations and risk of IPF: a meta-analysis and mRNA expression study. Immunogenetics 64(5):371-377
53. Xu D, Mu R, Wei X (2019) The roles of il-1 family cytokines in the pathogenesis of systemic sclerosis. Front Immunol 10:2025

54. Ahn MH et al (2011) A promoter SNP rs4073T $>$ A in the common allele of the interleukin 8 gene is associated with the development of idiopathic pulmonary fibrosis via the IL- 8 protein enhancing mode. Respir Res 12:73

55. Xue J et al (2011) The HLA class II Allele DRB1*1501 is overrepresented in patients with idiopathic pulmonary fibrosis. PLOS ONE 6(2):e14715

56. Caliskan C et al (2020) Genetic variation in CCL18 gene influences CCL18 expression and correlates with survival in idiopathic pulmonary fibrosis-part B. J Clin Med 9(6):1993

57. Salazar G, Mayes MD (2015) Genetics, epigenetics, and genomics of systemic sclerosis. Rheum Dis Clin North Am 41(3):345-366

58. Johannson KA, Balmes JR, Collard HR (2015) Air pollution exposure: a novel environmental risk factor for interstitial lung disease? Chest 147(4):1161-1167

59. Macneal K, Schwartz DA (2012) The genetic and environmental causes of pulmonary fibrosis. Proc Am Thorac Soc 9(3):120-125

60. Kreuter $M$ et al (2016) Impact of comorbidities on mortality in patients with idiopathic pulmonary fibrosis. PLOS ONE 11(3): 0151425

61. Arase $Y$ et al (2008) Hepatitis C virus enhances incidence of idiopathic pulmonary fibrosis. World J Gastroenterol 14(38):5880-5886

62. Wootton SC et al (2011) Viral infection in acute exacerbation of idiopathic pulmonary fibrosis. Am J Respir Crit Care Med 183(12):1698-1702

63. McDonald LT (2021) Healing after COVID-19: are survivors at risk for pulmonary fibrosis? Am J Physiol Lung Cell Mol Physiol 320(2):L257-L265

64. Hogan BL et al (2014) Repair and regeneration of the respiratory system: complexity, plasticity, and mechanisms of lung stem cell function. Cell Stem Cell 15(2):123-138

65. Nabhan AN et al (2018) Single-cell Wnt signaling niches maintain stemness of alveolar type 2 cells. Science 359(6380):1118-1123

66. Desai TJ, Brownfield DG, Krasnow MA (2014) Alveolar progenitor and stem cells in lung development, renewal and cancer. Nature 507(7491):190-194

67. Zacharias WJ et al (2018) Regeneration of the lung alveolus by an evolutionarily conserved epithelial progenitor. Nature 555(7695):251-255

68. Selman M, Pardo A (2006) Role of epithelial cells in idiopathic pulmonary fibrosis: from innocent targets to serial killers. Proc Am Thorac Soc 3(4):364-372

69. Barkauskas CE et al (2013) Type 2 alveolar cells are stem cells in adult lung. J Clin Invest 123(7):3025-3036

70. $\mathrm{Xu} \mathrm{Y}$ et al (2016) Single-cell RNA sequencing identifies diverse roles of epithelial cells in idiopathic pulmonary fibrosis. JCI Insight 1(20):e90558

71. Sisson TH et al (2010) Targeted injury of type II alveolar epithelial cells induces pulmonary fibrosis. Am J Respir Crit Care Med 181(3):254-263

72. Yao C et al (2020) Senescence of alveolar type 2 cells drives progressive pulmonary fibrosis. Am J Respir Crit Care Med 203:707

73. Urbanczik R (1988) Problems in relation to chemotherapy of mycobacterioses caused by so-called atypical mycobacteria. Prax Klin Pneumol 42(7):610-612

74. Chen X et al (2019) Epithelial cell senescence induces pulmonary fibrosis through Nanog-mediated fibroblast activation. Aging (Albany NY) 12(1):242-259

75. Tian Y et al (2019) Loss of PTEN induces lung fibrosis via alveolar epithelial cell senescence depending on NF-kappaB activation. Aging Cell 18(1):e12858 
76. Minagawa $S$ et al (2011) Accelerated epithelial cell senescence in IPF and the inhibitory role of SIRT6 in TGF-beta-induced senescence of human bronchial epithelial cells. Am J Physiol Lung Cell Mol Physiol 300(3):L391-401

77. Zhao L, Yee M, O'Reilly MA (2013) Transdifferentiation of alveolar epithelial type II to type I cells is controlled by opposing TGF-beta and BMP signaling. Am J Physiol Lung Cell Mol Physiol 305(6):L409-L418

78. Finn J et al (2019) Dlk1-mediated temporal regulation of notch signaling is required for differentiation of alveolar type II to type I cells during repair. Cell Rep 26(11):2942-2954

79. Strunz M et al (2020) Alveolar regeneration through a Krt8+ transitional stem cell state that persists in human lung fibrosis. Nat Commun 11(1):3559

80. Adams TS et al (2020) Single-cell RNA-seq reveals ectopic and aberrant lung-resident cell populations in idiopathic pulmonary fibrosis. Sci Adv 6(28):eaba1983

81. Kim KK et al (2006) Alveolar epithelial cell mesenchymal transition develops in vivo during pulmonary fibrosis and is regulated by the extracellular matrix. Proc Natl Acad Sci USA 103(35):13180-13185

82. Willis BC et al (2005) Induction of epithelial-mesenchymal transition in alveolar epithelial cells by transforming growth factor-beta1: potential role in idiopathic pulmonary fibrosis. Am J Pathol 166(5):1321-1332

83. Willis BC, Borok Z (2007) TGF-beta-induced EMT: mechanisms and implications for fibrotic lung disease. Am J Physiol Lung Cell Mol Physiol 293(3):L525-L534

84. Rock JR et al (2011) Multiple stromal populations contribute to pulmonary fibrosis without evidence for epithelial to mesenchymal transition. Proc Natl Acad Sci USA 108(52):E1475-E1483

85. Korpal $\mathrm{M}$ et al (2008) The miR-200 family inhibits epithelialmesenchymal transition and cancer cell migration by direct targeting of E-cadherin transcriptional repressors ZEB1 and ZEB2. J Biol Chem 283(22):14910-14914

86. Gregory PA et al (2008) The miR-200 family and miR-205 regulate epithelial to mesenchymal transition by targeting ZEB1 and SIP1. Nat Cell Biol 10(5):593-601

87. Pandit KV et al (2010) Inhibition and role of let-7d in idiopathic pulmonary fibrosis. Am J Respir Crit Care Med 182(2):220-229

88. Pandit KV, Milosevic J, Kaminski N (2011) MicroRNAs in idiopathic pulmonary fibrosis. Transl Res 157(4):191-199

89. Mescher AL (2017) Macrophages and fibroblasts during inflammation and tissue repair in models of organ regeneration. Regeneration (Oxf) 4(2):39-53

90. Kliment CR, Oury TD (2010) Oxidative stress, extracellular matrix targets, and idiopathic pulmonary fibrosis. Free Radic Biol Med 49(5):707-717

91. Fois AG et al (2018) Evaluation of oxidative stress biomarkers in idiopathic pulmonary fibrosis and therapeutic applications: a systematic review. Respir Res 19(1):51

92. Paliogiannis $\mathrm{P}$ et al (2018) Oxidative stress-linked biomarkers in idiopathic pulmonary fibrosis: a systematic review and metaanalysis. Biomark Med 12(10):1175-1184

93. Liu RM, Gaston Pravia KA (2010) Oxidative stress and glutathione in TGF-beta-mediated fibrogenesis. Free Radic Biol Med 48(1):1-15

94. Hecker L et al (2009) NADPH oxidase-4 mediates myofibroblast activation and fibrogenic responses to lung injury. Nat Med 15(9):1077-1081

95. Bernard K et al (2017) NADPH oxidase 4 (Nox4) suppresses mitochondrial biogenesis and bioenergetics in lung fibroblasts via a nuclear factor erythroid-derived 2-like 2 (Nrf2)-dependent pathway. J Biol Chem 292(7):3029-3038

96. Dasari A et al (2006) Oxidative stress induces premature senescence by stimulating caveolin-1 gene transcription through p38 mitogen-activated protein kinase/Sp1-mediated activation of two GC-rich promoter elements. Cancer Res 66(22):10805-10814

97. Campisi J, di Fagagna Fd'A (2007) Cellular senescence: when bad things happen to good cells. Nat Rev Mol Cell Biol 8(9):729-40

98. Toussaint O, Medrano EE, von Zglinicki T (2000) Cellular and molecular mechanisms of stress-induced premature senescence (SIPS) of human diploid fibroblasts and melanocytes. Exp Gerontol 35(8):927-945

99. $\mathrm{Ng} \mathrm{IH}$ et al (2014) Oxidative stress impairs multiple regulatory events to drive persistent cytokine-stimulated STAT3 phosphorylation. Biochim Biophys Acta 1843(3):483-494

100. Moodley YP et al (2003) Inverse effects of interleukin-6 on apoptosis of fibroblasts from pulmonary fibrosis and normal lungs. Am J Respir Cell Mol Biol 29(4):490-498

101. Yu G et al (2018) Thyroid hormone inhibits lung fibrosis in mice by improving epithelial mitochondrial function. Nat Med 24(1):39-49

102. Mostmans $Y$ et al (2017) The role of endothelial cells in the vasculopathy of systemic sclerosis: a systematic review. Autoimmun Rev 16(8):774-786

103. Jimenez SA, Piera-Velazquez S (2016) Endothelial to mesenchymal transition (EndoMT) in the pathogenesis of systemic sclerosis-associated pulmonary fibrosis and pulmonary arterial hypertension. Myth or reality? Matrix Biol 51:26-36

104. Jain M et al (2013) Mitochondrial reactive oxygen species regulate transforming growth factor-beta signaling. J Biol Chem 288(2):770-777

105. Evrard SM et al (2016) Endothelial to mesenchymal transition is common in atherosclerotic lesions and is associated with plaque instability. Nat Commun 7:11853

106. $\mathrm{Ma} \mathrm{Z}$ et al (2017) Lovastatin alleviates endothelial-to-mesenchymal transition in glomeruli via suppression of oxidative stress and TGF-beta1 signaling. Front Pharmacol 8:473

107. Medici D, Potenta S, Kalluri R (2011) Transforming growth factor-beta2 promotes snail-mediated endothelial-mesenchymal transition through convergence of smad-dependent and smadindependent signalling. Biochem J 437(3):515-520

108. Goumans MJ, Liu Z, ten Dijke P (2009) TGF-beta signaling in vascular biology and dysfunction. Cell Res 19(1):116-127

109. Piera-Velazquez S, Jimenez SA (2012) Molecular mechanisms of endothelial to mesenchymal cell transition (EndoMT) in experimentally induced fibrotic diseases. Fibrogenesis Tissue Repair 5(Suppl 1):S7

110. Vandewalle C, Van Roy F, Berx G (2009) The role of the ZEB family of transcription factors in development and disease. Cell Mol Life Sci 66(5):773-787

111. Wermuth PJ et al (2016) Stimulation of transforming growth factor-beta1-induced endothelial-to-mesenchymal transition and tissue fibrosis by endothelin-1 (ET-1): a novel profibrotic effect of ET-1. PLOS ONE 11(9):e0161988

112. Thuan DTB et al (2018) A potential link between oxidative stress and endothelial-to-mesenchymal transition in systemic sclerosis. Front Immunol 9:1985

113. Del Galdo F et al (2008) Decreased expression of caveolin 1 in patients with systemic sclerosis: crucial role in the pathogenesis of tissue fibrosis. Arthritis Rheum 58(9):2854-2865

114. Tourkina E et al (2008) Antifibrotic properties of caveolin-1 scaffolding domain in vitro and in vivo. Am J Physiol Lung Cell Mol Physiol 294(5):L843-L861

115. Wang XM et al (2006) Caveolin-1: a critical regulator of lung fibrosis in idiopathic pulmonary fibrosis. J Exp Med 203(13):2895-2906

116. Fu Y et al (2009) Differential regulation of transforming growth factor beta signaling pathways by notch in human endothelial cells. J Biol Chem 284(29):19452-19462 
117. Wei $\mathbf{J}$ et al (2012) Wnt/beta-catenin signaling is hyperactivated in systemic sclerosis and induces smad-dependent fibrotic responses in mesenchymal cells. Arthritis Rheum 64(8):2734-2745

118. Bhattacharyya $\mathrm{S}$ et al (2018) TLR4-dependent fibroblast activation drives persistent organ fibrosis in skin and lung. JCI Insight. https://doi.org/10.1172/jci.insight.98850

119. Bhattacharyya $S$ et al (2016) Tenascin-C drives persistence of organ fibrosis. Nat Commun 7:11703

120. Bhattacharyya $S$ et al (2014) FibronectinEDA promotes chronic cutaneous fibrosis through Toll-like receptor signaling. Sci Transl Med 6(232):232ra50

121. Novi F et al (2004) The paired activation of the two components of the muscarinic M3 receptor dimer is required for induction of ERK1/2 phosphorylation. J Biol Chem 279(9):7476-7486

122. Li LC, Gao J, Li J (2014) Emerging role of HMGB1 in fibrotic diseases. J Cell Mol Med 18(12):2331-2339

123. Jiang D et al (2005) Regulation of lung injury and repair by Tolllike receptors and hyaluronan. Nat Med 11(11):1173-1179

124. Ah Kioon, M.D., et al., Plasmacytoid dendritic cells promote systemic sclerosis with a key role for TLR8. Sci Transl Med, 2018. 10(423).

125. Fang F et al (2016) Toll-like receptor 9 signaling is augmented in systemic sclerosis and elicits transforming growth factor beta-dependent fibroblast activation. Arthritis Rheumatol 68(8):1989-2002

126. Artlett $\mathrm{CM}$ et al (2011) The inflammasome activating caspase 1 mediates fibrosis and myofibroblast differentiation in systemic sclerosis. Arthritis Rheum 63(11):3563-3574

127. Denton CP et al (2018) Therapeutic interleukin-6 blockade reverses transforming growth factor-beta pathway activation in dermal fibroblasts: insights from the fasscinate clinical trial in systemic sclerosis. Ann Rheum Dis 77(9):1362-1371

128. Epstein Shochet G et al (2020) TGF-beta pathway activation by idiopathic pulmonary fibrosis (IPF) fibroblast derived soluble factors is mediated by IL-6 trans-signaling. Respir Res 21(1):56

129. Khanna D et al (2018) Safety and efficacy of subcutaneous tocilizumab in systemic sclerosis: results from the open-label period of a phase II randomised controlled trial (fasscinate). Ann Rheum Dis 77(2):212-220

130. Castelino FV et al (2016) An Autotaxin/lysophosphatidic acid/ interleukin-6 amplification loop drives scleroderma fibrosis. Arthritis Rheumatol 68(12):2964-2974

131. Reyfman PA et al (2019) Single-cell transcriptomic analysis of human lung provides insights into the pathobiology of pulmonary fibrosis. Am J Respir Crit Care Med 199(12):1517-1536

132. Duru N, Wolfson B, Zhou Q (2016) Mechanisms of the alternative activation of macrophages and non-coding RNAs in the development of radiation-induced lung fibrosis. World J Biol Chem 7(4):231-239

133. Allanore $\mathrm{Y}$ et al (2020) A randomised, double-blind, placebo-controlled, 24-week, phase II, proof-of-concept study of romilkimab (SAR156597) in early diffuse cutaneous systemic sclerosis. Ann Rheum Dis 79(12):1600-1607

134. Pillai S (2019) $\mathrm{T}$ and B lymphocytes in fibrosis and systemic sclerosis. Curr Opin Rheumatol 31(6):576-581

135. Kowal-Bielecka O et al (2017) Update of EULAR recommendations for the treatment of systemic sclerosis. Ann Rheum Dis 76(8):1327-1339

136. Volkmann ER et al (2019) Cyclophosphamide for systemic sclerosis-related interstitial lung disease: a comparison of scleroderma lung study I and II. J Rheumatol 46(10):1316-1325

137. Mattoo $\mathrm{H}$ et al (2016) Clonal expansion of CD4(+) cytotoxic $\mathrm{T}$ lymphocytes in patients with IgG4-related disease. J Allergy Clin Immunol 138(3):825-838
138. Perugino CA et al (2021) CD4(+) and CD8(+) cytotoxic T lymphocytes may induce mesenchymal cell apoptosis in IgG4related disease. J Allergy Clin Immunol 147(1):368-382

139. Maehara $\mathrm{T}$ et al (2020) Cytotoxic CD4+ T lymphocytes may induce endothelial cell apoptosis in systemic sclerosis. J Clin Invest 130(5):2451-2464

140. Allard-Chamard $\mathrm{H}$ et al (2021) CD4(+)CTLs in fibrosing mediastinitis linked to histoplasma capsulatum. J Immunol 206(3):524-530

141. Taylor DK et al (2018) T follicular helper-like cells contribute to skin fibrosis. Sci Transl Med 10(431):eaaf5307

142. Ricard L et al (2019) Circulating follicular helper $\mathrm{T}$ cells are increased in systemic sclerosis and promote plasmablast differentiation through the IL-21 pathway which can be inhibited by ruxolitinib. Ann Rheum Dis 78(4):539-550

143. Korman B (2019) Evolving insights into the cellular and molecular pathogenesis of fibrosis in systemic sclerosis. Transl Res 209:77-89

144. El Agha E et al (2017) Two-way conversion between lipogenic and myogenic fibroblastic phenotypes marks the progression and resolution of lung fibrosis. Cell Stem Cell 20(2):261-273

145. Henderson NC et al (2013) Targeting of alphav integrin identifies a core molecular pathway that regulates fibrosis in several organs. Nat Med 19(12):1617-1624

146. Xie $\mathrm{T}$ et al (2016) Transcription factor $\mathrm{TBX} 4$ regulates myofibroblast accumulation and lung fibrosis. J Clin Invest 126(8):3063-3079

147. Dulauroy $\mathrm{S}$ et al (2012) Lineage tracing and genetic ablation of ADAM12(+) perivascular cells identify a major source of profibrotic cells during acute tissue injury. Nat Med 18(8):1262-1270

148. Habiel DM, Hogaboam CM (2017) Heterogeneity of fibroblasts and myofibroblasts in pulmonary fibrosis. Curr Pathobiol Rep 5(2):101-110

149. Booth AJ et al (2012) Acellular normal and fibrotic human lung matrices as a culture system for in vitro investigation. Am J Respir Crit Care Med 186(9):866-876

150. Parker MW et al (2014) Fibrotic extracellular matrix activates a profibrotic positive feedback loop. J Clin Invest 124(4):1622-1635

151. Angelidis I et al (2019) An atlas of the aging lung mapped by single cell transcriptomics and deep tissue proteomics. Nat Commun 10(1):963

152. Burgstaller $\mathrm{G}$ et al (2017) The instructive extracellular matrix of the lung: basic composition and alterations in chronic lung disease. Eur Respir J 50(1):1601805

153. Ahrman E et al (2018) Quantitative proteomic characterization of the lung extracellular matrix in chronic obstructive pulmonary disease and idiopathic pulmonary fibrosis. J Proteomics 189:23-33

154. Tschumperlin DJ et al (2018) Mechanosensing and fibrosis. J Clin Invest 128(1):74-84

155. Haak AJ et al (2019) Selective YAP/TAZ inhibition in fibroblasts via dopamine receptor D1 agonism reverses fibrosis. Sci Transl Med 11(516):eaau6296

156. Moore BB et al (2013) Animal models of fibrotic lung disease. Am J Respir Cell Mol Biol 49(2):167-179

157. Yu G, Ibarra GH, Kaminski N (2018) Fibrosis: lessons from OMICS analyses of the human lung. Matrix Biol 68-69:422-434

158. Kaminski N, Rosas IO (2006) Gene expression profiling as a window into idiopathic pulmonary fibrosis pathogenesis: can we identify the right target genes? Proc Am Thorac Soc 3(4):339-344

159. Selman M, Pardo A, Kaminski N (2008) Idiopathic pulmonary fibrosis: aberrant recapitulation of developmental programs? PLoS Med 5(3):e62 
160. Bueno $\mathrm{M}$ et al (2015) PINK1 deficiency impairs mitochondrial homeostasis and promotes lung fibrosis. J Clin Invest 125(2):521-538

161. Yang IV et al (2013) Expression of cilium-associated genes defines novel molecular subtypes of idiopathic pulmonary fibrosis. Thorax 68(12):1114-1121

162. Selman $M$ et al (2006) Gene expression profiles distinguish idiopathic pulmonary fibrosis from hypersensitivity pneumonitis. Am J Respir Crit Care Med 173(2):188-198

163. Montoro DT et al (2018) A revised airway epithelial hierarchy includes CFTR-expressing ionocytes. Nature 560(7718):319-324

164. Plasschaert LW et al (2018) A single-cell atlas of the airway epithelium reveals the CFTR-rich pulmonary ionocyte. Nature 560(7718):377-381

165. Regev A et al (2017) The human cell atlas. Elife 6:e27041

166. Morse $\mathrm{C}$ et al (2019) Proliferating SPP1/MERTK-expressing macrophages in idiopathic pulmonary fibrosis. Eur Respir J 54(2): 1802441

167. Distler O et al (2019) Nintedanib for systemic sclerosis-associated interstitial lung disease. N Engl J Med 380(26):2518-2528

168. Del Papa N et al (2018) Autologous hematopoietic stem cell transplantation for treatment of systemic sclerosis. Front Immunol 9:2390

169. Noth I et al (2012) A placebo-controlled randomized trial of warfarin in idiopathic pulmonary fibrosis. Am J Respir Crit Care Med 186(1):88-95

170. Richeldi L et al (2014) Efficacy and safety of nintedanib in idiopathic pulmonary fibrosis. N Engl J Med 370(22):2071-2082

171. King TE Jr et al (2014) A phase 3 trial of pirfenidone in patients with idiopathic pulmonary fibrosis. N Engl J Med 370(22):2083-2092

172. Noble PW et al (2011) Pirfenidone in patients with idiopathic pulmonary fibrosis (CAPACITY): two randomised trials. Lancet 377(9779):1760-1769

173. Raghu $\mathrm{G}$ et al (2015) CC-chemokine ligand 2 inhibition in idiopathic pulmonary fibrosis: a phase 2 trial of carlumab. Eur Respir J 46(6): 1740-1750

174. Raghu $\mathrm{G}$ et al (2008) Treatment of idiopathic pulmonary fibrosis with etanercept: an exploratory, placebo-controlled trial. Am J Respir Crit Care Med 178(9):948-955

175. Raghu $G$ et al (2017) Efficacy of simtuzumab versus placebo in patients with idiopathic pulmonary fibrosis: a randomised, double-blind, controlled, phase 2 trial. Lancet Respir Med 5(1):22-32

176. Parker JM et al (2018) A phase 2 randomized controlled study of tralokinumab in subjects with idiopathic pulmonary fibrosis. Am J Respir Crit Care Med 197(1):94-103
177. Richeldi L et al (2020) Pamrevlumab, an anti-connective tissue growth factor therapy, for idiopathic pulmonary fibrosis (PRAISE): a phase 2, randomised, double-blind, placebo-controlled trial. Lancet Respir Med 8(1):25-33

178. Maher TM et al (2018) Safety, tolerability, pharmacokinetics, and pharmacodynamics of GLPG1690, a novel autotaxin inhibitor, to treat idiopathic pulmonary fibrosis (FLORA): a phase 2a randomised placebo-controlled trial. Lancet Respir Med 6(8):627-635

179. Moreira, A.P., et al., Serum amyloid $P$ attenuates $M 2$ macrophage activation and protects against fungal spore-induced allergic airway disease. J Allergy Clin Immunol, 2010. 126(4): p. 712-721 e7.

180. Murray LA et al (2011) TGF-beta driven lung fibrosis is macrophage dependent and blocked by Serum amyloid P. Int J Biochem Cell Biol 43(1):154-162

181. Raghu $\mathrm{G}$ et al (2018) Effect of recombinant human pentraxin 2 vs placebo on change in forced vital capacity in patients with idiopathic pulmonary fibrosis: a randomized clinical trial. JAMA 319(22):2299-2307

182. Raghu $G$ et al (2019) Long-term treatment with recombinant human pentraxin 2 protein in patients with idiopathic pulmonary fibrosis: an open-label extension study. Lancet Respir Med 7(8):657-664

183. Tashkin DP et al (2016) Mycophenolate mofetil versus oral cyclophosphamide in scleroderma-related interstitial lung disease (SLS II): a randomised controlled, double-blind, parallel group trial. Lancet Respir Med 4(9):708-719

184. Fernandez-Codina A et al (2018) Treatment algorithms for systemic sclerosis according to experts. Arthritis Rheumatol 70(11):1820-1828

185. Elhai M et al (2019) Outcomes of patients with systemic sclerosis treated with rituximab in contemporary practice: a prospective cohort study. Ann Rheum Dis 78(7):979-987

186. Daoussis D et al (2017) A multicenter, open-label, comparative study of B cell depletion therapy with rituximab for systemic sclerosis-associated interstitial lung disease. Semin Arthritis Rheum 46(5):625-631

187. Gordon JK et al (2018) Belimumab for the treatment of early diffuse systemic sclerosis: results of a randomized, double-blind, placebo-controlled pilot trial. Arthritis Rheumatol 70(2):308-316

Publisher's Note Springer Nature remains neutral with regard to jurisdictional claims in published maps and institutional affiliations. 